Proceedings of the XXXVIII International School and Conference on the Physics of Semiconductors "Jaszowiec" 2009

\title{
Magnetization Dynamics of a (Cd,Mn)Te Quantum Well in Pulsed Magnetic Field
}

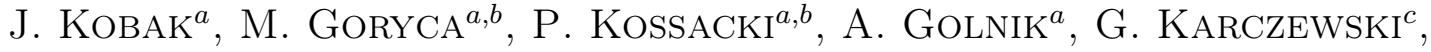 \\ T. WoJTOWICZ ${ }^{c}$ AND J.A. GAJ ${ }^{a}$ \\ ${ }^{a}$ Institute of Experimental Physics, University of Warsaw, Hoża 69, 00-681 Warsaw, Poland \\ ${ }^{b}$ Grenoble High Magnetic Field Laboratory, CNRS, Grenoble, France \\ ${ }^{c}$ Institute of Physics, Polish Academy of Sciences, Warsaw, Poland
}

In this paper we present studies of magnetization relaxation in a $(\mathrm{Cd}, \mathrm{Mn}) \mathrm{Te}$ quantum well containing $3.2 \%$ of $\mathrm{Mn}$, after a pulse of magnetic field. The relaxation was found to be very fast, with dominant component faster than $10 \mathrm{~ns}$. Upon application of static magnetic field the relaxation does not slow down, in contrast with the behavior of very diluted quantum wells or bulk material.

PACS numbers: 78.67.Hc, 71.70.Gm, 78.55.Et, 75.75.-c

\section{Introduction}

Magnetization dynamics in bulk (Cd,Mn)Te crystals has been studied by many authors since the eighties. It was demonstrated that in magnetic field of the order of $1 \mathrm{~T}$, the magnetization relaxation is mainly caused by the spin-lattice interaction $[1,2]$. Its characteristic time is of the order of microseconds for samples with Mn content equal to a few percent. Our present work is devoted to two-dimensional structures — quantum wells (QWs). In this paper we present results of magnetization relaxation studies of a $(\mathrm{Cd}, \mathrm{Mn}) \mathrm{Te} \mathrm{QW}$ in a pulsed magnetic field.

\section{Sample and experiment}

The sample used in the experiment was grown by molecular beam epitaxy on GaAs substrate. In order

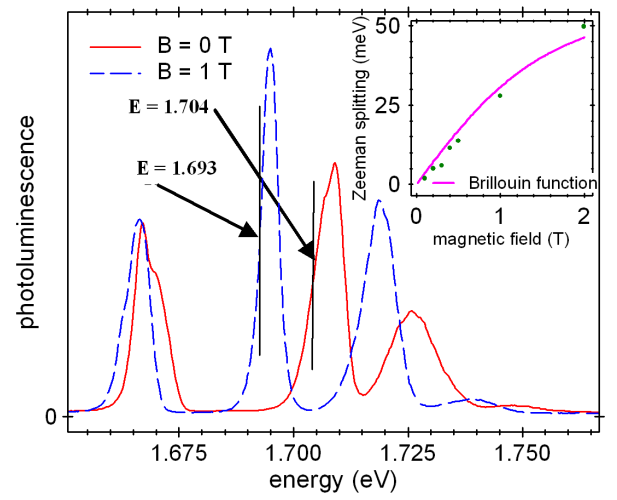

Fig. 1. Photoluminescence spectra of the studied sample for given values of the static magnetic field. Vertical lines indicate energies at which the temporal profiles of the photoluminescence were recorded upon application of the magnetic pulses. Inset: line position vs. magnetic field.

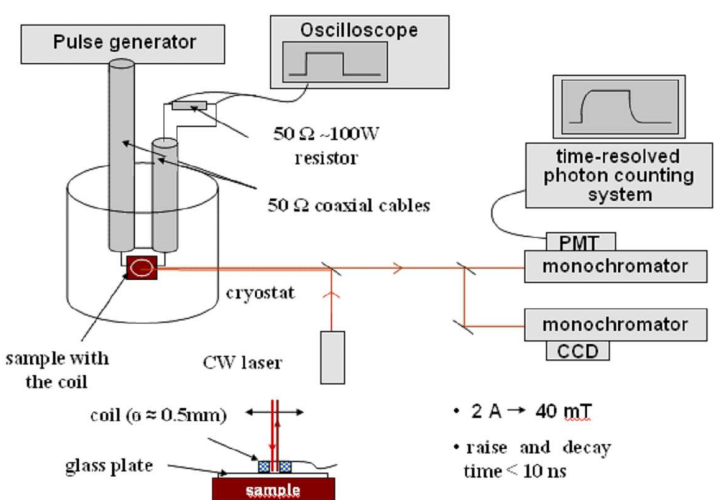

Fig. 2. Experimental setup.

to identify lines in the photoluminescence spectra of the sample we fit the Brillouin function to the dependence of the line positions versus magnetic field [3] (Fig. 1). The Mn content in the QW was equal to about $3.2 \%$ and the QW width was about $100 \AA$ while the Mn content in the barriers was equal to about $14 \%$. The measurements were performed at $1.58 \mathrm{~K}$ in the static magnetic field ranging from 0 to $2 \mathrm{~T}$. Additionally to the static magnetic field we applied pulsed field of amplitude equal to $40 \mathrm{mT}$ with raise and fall times shorter than $10 \mathrm{~ns}$. These pulses were produced with a small coil mounted directly on the surface of the sample (Fig. 2). Both fields - the static and the pulsed one - were applied in the Faraday configuration. The luminescence of the sample was excited with a $\mathrm{CW}$ laser, emitting at $680 \mathrm{~nm}$.

Light emitted by the sample was collected with a monochromator equipped either with a CCD camera or an avalanche photodiode connected to a time-resolved photon counting system. As a measure of the magnetization of the QW we used the Zeeman shift of the lumi- 
nescence line. The temporal profiles of the luminescence during and after the magnetic pulses were recorded at selected wavelengths on the slope of appropriate line [4].

\section{Results and discussion}

In absence of static magnetic field the magnetization relaxation after the magnetic pulse was found to be very fast, with a dominant component faster than $10 \mathrm{~ns}$ (see Fig. 3). This is similar to the results obtained for very diluted (Cd,Mn)Te QWs [5], for which the fast relaxation was caused mainly by the hyperfine interaction and strain.

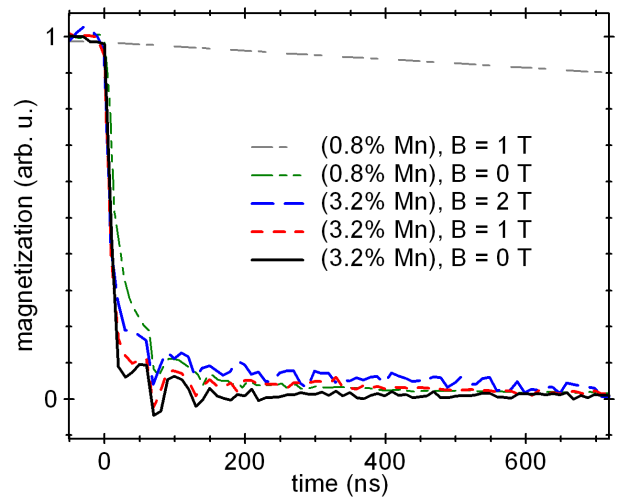

Fig. 3. The magnetization relaxation in indicated static magnetic fields, measured in (CdMn)Te QW containing $3.2 \% \mathrm{Mn}$, compared with the magnetization relaxation in very diluted (CdMn)Te QW (0.8\% Mn) [5].

Upon application of static magnetic field stronger than $1 \mathrm{~T}$, the magnetization relaxation in the currently studied sample does not slow down. This is different from the behavior observed for bulk and very diluted QWs, for which magnetic field switched off the relaxation channel of hyperfine interaction. As a result, the relaxation slowed down by 2 to 3 orders of magnitude. In the studied sample, the characteristic time of the dominant component of the relaxation remains below 10 ns. For higher fields, a weak slow component becomes also visible. To obtain its relative amplitude we fit a convolution of the temporal profile of the current in the coil with a bi-exponential decay to the temporal profile of the luminescence. The relative amplitude of the slow component rises with increasing magnetic field, as shown in Fig. 4. This remains in qualitative agreement with the behavior observed for very diluted (Cd,Mn)Te QWs [5], however the slow component is much weaker in currently studied sample and much higher magnetic field is needed to make this component visible.

This difference might be explained by interactions which mix spin states of Mn ions, other than hyperfine interaction. As shown in [5], a significant strain might be a cause of fast magnetization relaxation. Moreover, a large number of lattice defects are expected to be present in the studied sample. These defects can also play a role

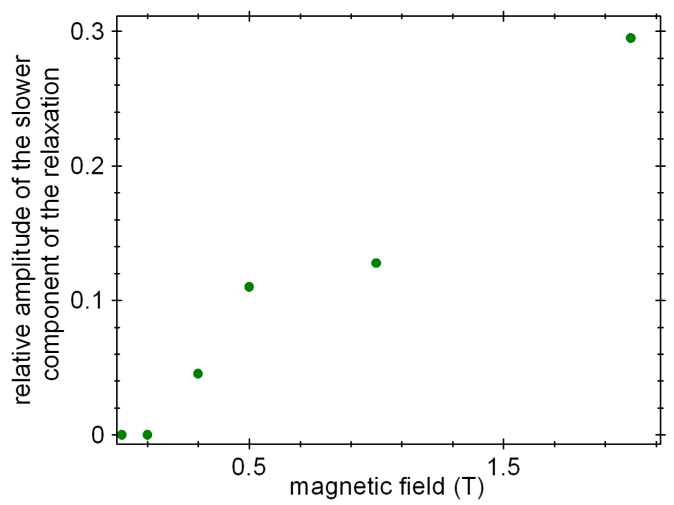

Fig. 4. Relative amplitude of the slower component of the magnetization relaxation vs. magnetic field.

of fast-relaxing centers, in particular for Mn content high enough to make spin-spin interaction an efficient channel of spin transfer towards these centers.

\section{Acknowledgments}

This work was partially supported by the Polish Ministry of Science and Higher Education as research grants in years 2006-2011, and by the 6th Research Framework Programme of EU (contract MTKD-CT-2005-029671).

\section{References}

[1] D. Scalbert, J. Cernogora, C. Benoît à la Guillaume, Solid State Commun. 66, 571 (1988).

[2] T. Strutz, A.M. Witowski, P. Wyder, Phys. Rev. Lett. 68, 3912 (1992).

[3] J.A. Gaj, W. Grieshaber, C. Bodin-Deshayes, J. Cibert, G. Feuillet, Y. Merle d'Aubigné, A. Wasiela, Phys. Rev. B 50, 5512 (1994).

[4] M. Goryca, D. Ferrand, P. Kossacki, M. Nawrocki, W. Pacuski, W. Maślana, S. Tatarenko, J. Cibert, Phys. Status Solidi B 243, 882 (2006).

[5] M. Goryca, D. Ferrand, P. Kossacki, M. Nawrocki, W. Pacuski, W. Maślana, J.A. Gaj, S. Tatarenko, J. Cibert, T. Wojtowicz, G. Karczewski, Phys. Rev. Lett. 102, 046408 (2009). 